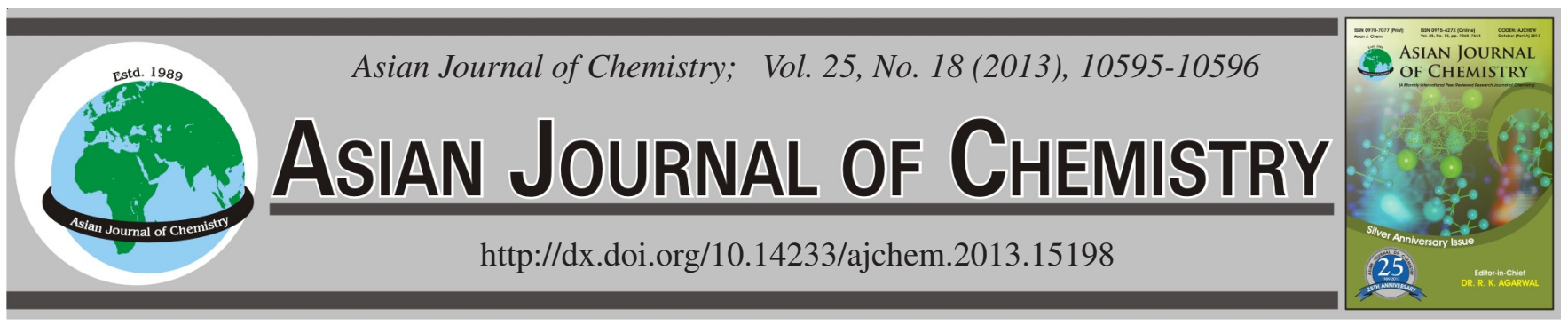

NOTE

\title{
Study on Novel Structure of Europium Complex
}

Hai-Xing Liu ${ }^{1, *}$, Qing-Hua Zhang ${ }^{1}$, Huan-Mei Guo ${ }^{1}$, Jing-Zhong Xiao,", Xi-Shi Tai ${ }^{1}$, Guang Zeng ${ }^{3}$ and Kai-Qi Ye ${ }^{4}$

${ }^{1}$ Chemistry \& Chemical and Environmental Engineering College, Weifang University, Weifang 261061, P.R. China

${ }^{2}$ CEMDRX, Department of Physics, University of Coimbra, Coimbra 3004-516, Portugal

${ }^{3}$ State Key Laboratory of Inorganic Synthesis and Preparative Chemistry, College of Chemistry, Jilin University, Changchun 130012, P.R. China

${ }^{4}$ State Key Laboratory of Supramolecular Structure and Materials, College of Chemistry, Jilin University, Changchun 130012, P.R. China

*Corresponding authors: E-mail: haixingliu@tom.com; jxiao@ua.pt

A novel europium complex with the m.f. $\mathrm{C}_{10} \mathrm{H}_{21} \mathrm{Eu}_{2} \mathrm{NO}_{19}$ has been synthesized from a hydrothermal reaction and the crystal structure has been determined by means of single-crystal X-ray diffraction. Europium atom is coordinated by nine $\mathrm{O}$ atoms. The molecular structure stabilized by the $\mathrm{O}-\mathrm{H} \cdots \mathrm{O}$ and $\mathrm{O}-\mathrm{H} \cdots \mathrm{N}$ hydrogen-bonding interactions.

Key Words: Europium complex, Structure analysis.

Lanthanide complexes have attracted attention of researchers for interesting structures and potential applications in luminescent materials, catalysts and magnetic molecular materials, etc. ${ }^{1-5}$. Among lanthanide ions, europium(III) ion has received the most attention because of good luminescence with narrow bandwidth, large stokes shift and long luminescence lifetime. The Eu complexes are successfully applied in materials science as luminescent probes and sensory materials ${ }^{6}$. carboxylates are often used to construct the coordination polymers because of their rich coordination modes ${ }^{7,8}$. A large number of lanthanide carboxylate complexes have been reported with dimeric or 1-, 2- and 3-D polymeric structures due to variable coordination number of $\mathrm{Ln}$ (III) as well as coordination versatility of carboxylate $e^{9-13}$. In this paper, the novel Eu complex is reported.

All commercially obtained reagent-grade chemicals were used without further purification. A mixture of $\mathrm{Eu}_{2} \mathrm{O}_{3}(0.01$ mmol, $0.004 \mathrm{~g}$ ), dilute $\mathrm{HNO}_{3}, 2$, 2'-bipyridine ( $0.1 \mathrm{mmol}, 0.016$ $\mathrm{g}), \mathrm{K}_{2} \mathrm{CrO}_{4}$ (0.1 mmol, $0.02 \mathrm{~g}$ ), boric acid (0.1 mmol, $0.006 \mathrm{~g}$ ) and tartaric acid $(0.2 \mathrm{mmol}, 0.03 \mathrm{~g})$ were added into $20 \mathrm{~mL}$ water with $10 \%(\mathrm{v} / \mathrm{v})$ ethanol and heated for $6 \mathrm{~h}$ at $413 \mathrm{~K}$. The solution was obtained by filtration after cooling the reaction to room temperature. Yellow block single crystals suitable for $\mathrm{X}$-ray measurements were obtained after a few weeks.

The crystal structure of title europium complex with the m.f. $\mathrm{C}_{10} \mathrm{H}_{21} \mathrm{Eu}_{2} \mathrm{NO}_{19}$ is shown in Fig. 1. The crystal data and structure refinement is shown in Table-1. The Eu atom is

\begin{tabular}{|c|c|}
\hline \multicolumn{2}{|c|}{$\begin{array}{c}\text { TABLE-1 } \\
\text { CRYSTAL DATA AND STRUCTURE } \\
\text { REFINEMENT FOR THE TITLE COMPLEX }\end{array}$} \\
\hline Empirical formula & $\mathrm{C}_{10} \mathrm{H}_{21} \mathrm{Eu}_{2} \mathrm{NO}_{19}$ \\
\hline Formula weight & 763.20 \\
\hline Temperature & 293(2) K \\
\hline Wavelength & $0.71073 \AA$ \\
\hline Crystal system, space group & Monoclinic, P2(1)/c \\
\hline Unit cell dimensions & $\begin{array}{l}\mathrm{a}=11.0821(12) \AA, \alpha=90^{\circ}, \\
\mathrm{b}=9.6205(9) \AA, \beta=114.204(2)^{\circ}, \\
\mathrm{c}=10.1103(10) \AA, \gamma=90^{\circ}\end{array}$ \\
\hline Volume & $983.15(17) \AA^{3}$ \\
\hline Z, Calculated density & $2,2.578 \mathrm{Mg} / \mathrm{m}^{3}$ \\
\hline Absorption coefficient & $6.421 \mathrm{~mm}^{-1}$ \\
\hline$F_{(000)}$ & 732 \\
\hline Crystal size & $0.43 \mathrm{~mm} \times 0.40 \mathrm{~mm} \times 0.39 \mathrm{~mm}$ \\
\hline Theta range for data collection & $3.06-25.02^{\circ}$ \\
\hline Limiting indices & $\begin{array}{l}-13 \Leftarrow \mathrm{h} \Leftarrow 13,-11 \Leftarrow \mathrm{k} \Leftarrow 11,-12 \\
\Leftarrow 1 \Leftarrow 12\end{array}$ \\
\hline Reflections collected/unique & $7439 / 1728\left[\mathrm{R}_{\text {(int) }}=0.0481\right]$ \\
\hline Completeness to theta $=25.02$ & $99.9 \%$ \\
\hline Absorption correction & Semi-empirical from equivalents \\
\hline Max. and min. transmission & 0.1885 and 0.1688 \\
\hline Refinement method & Full-matrix least-squares on $\mathrm{F}^{2}$ \\
\hline Data/restraints/parameters & $1728 / 0 / 175$ \\
\hline Goodness-of-fit on $\mathrm{F}^{2}$ & 1.064 \\
\hline Final $R$ indices $[I>2 \sigma(I)]$ & $\mathrm{R}_{1}=0.0317, \mathrm{wR}_{2}=0.0700$ \\
\hline $\mathrm{R}$ indices (all data) & $\mathrm{R}_{1}=0.0424, \mathrm{wR}_{2}=0.0783$ \\
\hline Largest diff. peak and hole & 2.731 and $-1.239 \mathrm{e} \AA^{-3}$ \\
\hline
\end{tabular}




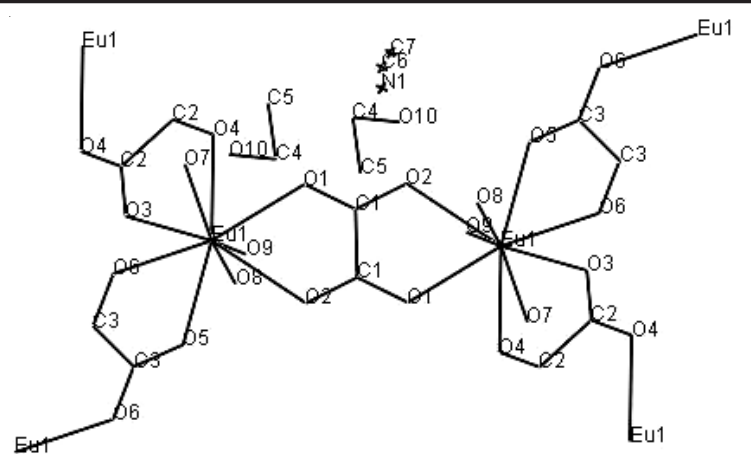

Fig. 1. Molecular structure of the Eu complex

coordinated by nine $\mathrm{O}$ atoms. One oxalic acid molecular bridges two Eu atoms. The Eu-O distances are in the range of 2.414-2.544 $\AA$. The angles of O-Eu-O are in the range of 63.98$143.8^{\circ}$. The torsion angles of $\mathrm{O}(1)-\mathrm{Eu}(1)-\mathrm{O}(3)-\mathrm{C}(2), \mathrm{O}(3)-$ $\mathrm{Eu}(1)-\mathrm{O}(1)-\mathrm{C}(1), \mathrm{O}(9)-\mathrm{Eu}(1)-\mathrm{O}(1)-\mathrm{C}(1)$ are 5.6, 129.1 and $64.0^{\circ}$, respectively. Selected bond lengths and bond angles are shown in Table-2.

\begin{tabular}{|c|c|c|c|}
\hline \multicolumn{4}{|c|}{$\begin{array}{c}\text { TABLE-2 } \\
\text { SELECT BOND LENGTHS }[\AA] \text { AND } \\
\text { ANGLES }\left[^{\circ}\right] \text { FOR THE TITLE COMPLEX }\end{array}$} \\
\hline $\mathrm{Eu}(1)-\mathrm{O}(5)$ & $2.414(5)$ & $\mathrm{O}(7)-\mathrm{Eu}(1)-\mathrm{O}(3)$ & $95.1(2)$ \\
\hline $\mathrm{Eu}(1)-\mathrm{O}(1)$ & $2.423(5)$ & $\mathrm{O}(9)-\mathrm{Eu}(1)-\mathrm{O}(3)$ & 71.84(19) \\
\hline $\mathrm{Eu}(1)-\mathrm{O}(7)$ & $2.423(6)$ & $\mathrm{O}(5)-\mathrm{Eu}(1)-\mathrm{O}(8)$ & $83.6(2)$ \\
\hline $\mathrm{Eu}(1)-\mathrm{O}(9)$ & $2.429(5)$ & $\mathrm{O}(1)-\mathrm{Eu}(1)-\mathrm{O}(8)$ & $81.0(2)$ \\
\hline $\mathrm{Eu}(1)-\mathrm{O}(3)$ & $2.464(5)$ & $\mathrm{O}(7)-\mathrm{Eu}(1)-\mathrm{O}(8)$ & $72.3(2)$ \\
\hline $\mathrm{Eu}(1)-\mathrm{O}(8)$ & $2.477(6)$ & $\mathrm{O}(9)-\mathrm{Eu}(1)-\mathrm{O}(8)$ & $137.3(2)$ \\
\hline $\mathrm{N}(1)-\mathrm{C}(6)$ & $1.11(4)$ & $\mathrm{C}(2)-\mathrm{O}(3)-\mathrm{Eu}(1)$ & $123.1(5)$ \\
\hline $\mathrm{O}(1)-\mathrm{C}(1)$ & $1.242(9)$ & $\mathrm{C}(3)-\mathrm{O}(5)-\mathrm{Eu}(1)$ & $121.4(5)$ \\
\hline $\mathrm{O}(2)-\mathrm{C}(1)$ & $1.263(9)$ & $\mathrm{O}(8)-\mathrm{Eu}(1)-\mathrm{O}(4) \# 3$ & $135.9(2)$ \\
\hline $\mathrm{O}(5)-\mathrm{C}(3)$ & $1.244(9)$ & $\mathrm{C}(1)-\mathrm{O}(1)-\mathrm{Eu}(1)$ & $121.2(5)$ \\
\hline $\mathrm{O}(6)-\mathrm{C}(3)$ & $1.257(9)$ & $\mathrm{C}(1)-\mathrm{O}(2)-\mathrm{Eu}(1) \# 1$ & $119.2(5)$ \\
\hline $\mathrm{O}(9)-\mathrm{H}(9 \mathrm{D})$ & 0.8501 & $\mathrm{C}(2)-\mathrm{O}(3)-\mathrm{Eu}(1)$ & $123.1(5)$ \\
\hline $\mathrm{O}(10)-\mathrm{C}(4)$ & $1.42(4)$ & $\mathrm{C}(2)-\mathrm{O}(4)-\mathrm{Eu}(1) \# 3$ & $120.1(5)$ \\
\hline $\mathrm{C}(4)-\mathrm{H}(4 \mathrm{~A})$ & 0.9700 & $\mathrm{C}(3)-\mathrm{O}(5)-\mathrm{Eu}(1)$ & $121.4(5)$ \\
\hline $\mathrm{C}(5)-\mathrm{H}(5 \mathrm{~B})$ & 0.9600 & $\mathrm{C}(3)-\mathrm{O}(6)-\mathrm{Eu}(1) \# 2$ & $119.2(5)$ \\
\hline$C(6)-C(7)$ & $1.43(5)$ & $\mathrm{Eu}(1)-\mathrm{O}(7)-\mathrm{H}(7 \mathrm{~F})$ & 134.6 \\
\hline $\mathrm{C}(7)-\mathrm{H}(7 \mathrm{C})$ & 0.9600 & $\mathrm{Eu}(1)-\mathrm{O}(7)-\mathrm{H}(7 \mathrm{G})$ & 116.2 \\
\hline $\mathrm{O}(5)-\mathrm{Eu}(1)-\mathrm{O}(1)$ & 137.48(19) & $\mathrm{H}(7 \mathrm{~F})-\mathrm{O}(7)-\mathrm{H}(7 \mathrm{G})$ & 108.7 \\
\hline $\mathrm{O}(5)-\mathrm{Eu}(1)-\mathrm{O}(7)$ & 137.06(19) & $\mathrm{Eu}(1)-\mathrm{O}(8)-\mathrm{H}(8 \mathrm{C})$ & 121.1 \\
\hline $\mathrm{O}(1)-\mathrm{Eu}(1)-\mathrm{O}(7)$ & 73.71(19) & $\mathrm{Eu}(1)-\mathrm{O}(8)-\mathrm{H}(8 \mathrm{D})$ & 128.6 \\
\hline $\mathrm{O}(5)-\mathrm{Eu}(1)-\mathrm{O}(9)$ & $76.1(2)$ & $\mathrm{H}(8 \mathrm{C})-\mathrm{O}(8)-\mathrm{H}(8 \mathrm{D})$ & 108.6 \\
\hline $\mathrm{O}(1)-\mathrm{Eu}(1)-\mathrm{O}(9)$ & $89.2(2)$ & $\mathrm{Eu}(1)-\mathrm{O}(9)-\mathrm{H}(9 \mathrm{C})$ & 118.2 \\
\hline $\mathrm{O}(7)-\mathrm{Eu}(1)-\mathrm{O}(9)$ & $143.8(2)$ & $\mathrm{O}(5)-\mathrm{C}(3)-\mathrm{O}(6)$ & $126.7(7)$ \\
\hline $\mathrm{O}(5)-\mathrm{Eu}(1)-\mathrm{O}(3)$ & $82.76(19)$ & $\mathrm{N}(1)-\mathrm{C}(6)-\mathrm{C}(7)$ & $167(4)$ \\
\hline $\mathrm{O}(1)-\mathrm{Eu}(1)-\mathrm{O}(3)$ & $130.50(19)$ & - & - \\
\hline
\end{tabular}

The molecular structure stabilized by the $\mathrm{O}-\mathrm{H} \cdots \mathrm{O}$ and $\mathrm{O}-\mathrm{H} \cdots \mathrm{N}$ hydrogen-bonding interactions.

\section{ACKNOWLEDGEMENTS}

This study were supported by the Natural Science Foundation of Shandong Province (No. ZR2010BL025), Open Project of State Key Laboratory of Supramolecular Structure and Materials (No. sklssm201323)(Jilin University), State Key Laboratory of Inorganic Synthesis and Preparative Chemistry (No. 2011-13) (Jilin University) and the National Science Foundation of China (No. 21171132).

\section{REFERENCES}

1. S.K. Ghosh, J.P. Zhang and S. Kitagawa, Angew. Chem., 119, 8111 (2007).

2. J.W. Han and C.L. Hill, J. Am. Chem. Soc., 129, 15094 (2007).

3. A. Fratini, G. Richards, E. Larder and S. Swavey, Inorg. Chem., 47, 1030 (2008).

4. B.D. Chandler, D.T. Cramb and G.K.H. Shimizu, J. Am. Chem. Soc., 128, 10403 (2006).

5. A. Mondry and P. Starynowicz, Eur. J. Inorg. Chem., 5, 1859 (2006).

6. J. Wang, P. Hu, B. Liu, R. Xu, X. Wang, D. Wang, L.Q. Zhang and X.D. Zhang, J. Struct. Chem., 52, 568 (2011).

7. X. Li, D.-Y. Wei, S.-J. Huang and Y.-Q. Zheng, J. Solid State Chem., 182, 95 (2009).

8. W. Li, X.-L. Wang, X.-Y. Song, L.-C. Li, D.-Z. Liao and Z.-H. Jiang, J. Mol. Struct., 885, 1 (2008).

9. U.P. Singh, R. Kumar and S. Upreti, J. Mol. Struct., 831, 97 (2007).

10. X. Li, Z.Y. Zhang, T.T. Zhang and Y.L. Ju, J. Coord. Chem., 60, 301 (2007).

11. X. Li, T.T. Zhang, Y.L. Ju, C.Y. Wang, Y.Q. Li, L. Zhang and Q. Zhang, J. Coord. Chem., 60, 2121 (2007).

12. X. Li, Y.L. Ju and Y.Q. Li, J. Coord. Chem., 61, 692 (2008).

13. X.-S. Wu, Y.-B. Zhang, X. Li and P.-Z. Li, J. Coord. Chem., 62, 797 (2009). 